\title{
LATTICE FUNCTION MEASUREMENTS IN THE FERMILAB ACCUMULATOR RING
}

\author{
M. Church, Fermilab*, PO Box 500 Batavia, IL, 60510
}

\section{Abstract}

The betatron phase advance between two points in a storage ring can be very accurately determined from a transverse beam transfer function measurement with DC beam. The phase advance can be determined from the difference in the phase response between an upper betatron sideband (N+Q line) and a lower betatron sideband (N-Q line) in an open loop network analyzer measurement between a kicker and a pickup. The beta functions can then be extracted from these phase measurements. This method has the advantage that it does not depend on the amplitude response of the pickup, kicker, or electronics. This technique is applied to the Fermilab Accumulator Ring to measure the betatron phase advance at 90 points around the ring, and these measurements are compared to a MAD[1] model.

\section{INTRODUCTION}

The Accumulator was designed to accumulate and store antiprotons for use in Fermilab's Collider program[2]. Relevant machine parameters are listed in Table 1.

\begin{tabular}{|c|c|}
\hline central momentum & $8820 \mathrm{MeV} / \mathrm{c}$ \\
\hline revolution period & $628820 . \mathrm{Hz}$ \\
\hline circumference & 474 meters \\
\hline gammaT & 5.45 \\
\hline horizontal tune & 6.609 \\
\hline vertical tune & 8.607 \\
\hline symmetry & $\begin{array}{c}\text { 3-fold symmetric; } \\
\text { each section mirror } \\
\text { symmetric }\end{array}$ \\
\hline
\end{tabular}

Table 1: Accumulator parameters

It has been in operation since 1985 and there has never been an accurate experimental determination of the lattice parameters. Recent phasing measurements with the ring's stochastic cooling systems indicate there is a discrepancy between the design lattice and the actual lattice. It is difficult to fully characterize the lattice from the standard technique of adjusting a quadrupole strength and observing the subsequent tune shift because only 9 of the 84 quadrupoles are instrumented with separately controllable shunt circuits. Measuring the beta functions by the

\footnotetext{
* Operated by Universities Research Association, Inc. under contract with the U.S Department of Energy.
}

method of "1-bumps" is limited by the uncertainty in the BPM calibration (greater than 10\%). In addition, measuring phase advance via turn-by-turn oscillations from a one-pass kick is only possible on the injection/extraction orbit of the machine and is limited by signal/noise in the BPM's. In the Accumulator, measuring the phase advance by comparing phase response difference between upper and lower Schottky sidebands offers the greatest potential for accurately determining the lattice.

The amplitude response of a beam to a transverse excitation at frequency $\omega$ has been calculated by several authors[3][4]. It is given by:

$$
\begin{aligned}
& \frac{V_{\text {out }}(\omega)}{V_{\text {in }}(\omega)}=-i P(\omega) \int \Psi\left(\omega^{\prime}\right) \frac{\sqrt{\beta_{P U} \beta_{K}}}{4} e^{i 2 \pi \alpha \omega\left(\frac{1}{\omega^{\prime}}-\frac{1}{\omega_{c}}\right)} \\
& {\left[e^{i 2 \pi \alpha_{2} Q\left(1+\frac{i}{\tan \left(\frac{\pi \omega}{\omega^{\prime}}+\pi Q\right)}\right)}\right.} \\
& \left.-e^{-i 2 \pi \alpha_{2} Q}\left(1+\frac{i}{\tan \left(\frac{\pi \omega}{\omega^{\prime}}-\pi Q\right)}\right)\right] d \omega^{\prime}
\end{aligned}
$$

where $\omega$ is the frequency of excitation, $P(\omega)$ contains the pickup, kicker, and electronic response functions, $\Psi\left(\omega^{\prime}\right)$ is the beam distribution, $\beta$ are the beta functions at the pickup and kicker, $Q$ is the tune, $\alpha$ is the fractional distance from pickup to kicker, $\alpha_{2}$ is the fractional phase advance from pickup to kicker, and $\omega_{c}$ is the frequency at which the cable delay is matched to the beam delay. At harmonics with no Schottky band overlap, the terms in brackets in eq. (1) separate into a single pole at each sideband. For the case of a symmetric beam distribution, 0 chromaticity, $\omega_{c}$ set to the central beam frequency, no Schottky band overlap, and if $P(\omega)$ has a linear phase slope, the response at the center of the sidebands is dominated by the residue term in the integral. The difference in phase between the upper sideband (USB) response and the lower sideband (LSB) response reduces to

$$
\phi_{U S B}-\phi_{L S B}=2\left((2 n+1) \frac{\pi}{2}-\phi_{P U-K}\right)
$$

where $\mathrm{n}$ is an integer and $\phi_{P U-K}$ is the betatron phase advance from the pickup to the kicker. 


\section{MEASUREMENT}

The measurement setup is shown in Figure 1. The pickups used were the Accumulator BPM pickups[5], which are split plate capacitive pickups. The kickers used were the Accumulator damper kickers[6], which are stripline kickers terminated in $50 \Omega$. Cables used were 3/8" and 1/2" heliax (1500-2400 nsec) and RG58 (40-400 nsec). Additional electrical delay was added in the network analyzer to make the total electrical delay equal to the beam delay to within a few 10's of nsec in order to avoid any confusion with 360 degree phase wrap. The deviation

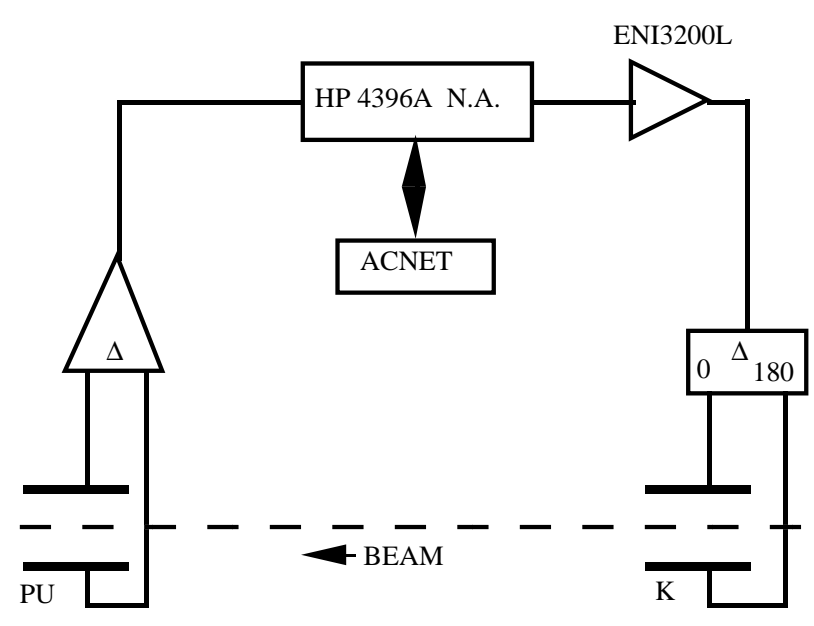

Figure 1: Measurement setup

of the electronic phase slope from linear was determined to be less than 1 degree over the ranges of interest by making $\mathrm{S}_{21}$ measurements of the input and output sections of the loop using the damper kicker fanback signal and the BPM test signal cable. An application program was written to interface the accelerator controls system to the network analyzer and to record data to disk for later analysis.

For the measurement $3 \times 10^{10}$ antiprotons were stored on the "core" orbit of the Accumulator and stochastically cooled in all 3 dimensions to transverse emittances of about $1 \pi$-mm-mrad and a momentum full width of $4 \mathrm{MeV} / \mathrm{c}$. Tunes were separated slightly to .610 and .604 to avoid confusion between horizontal and vertical measurements. The chromaticity was measured to be $\leq .5$ in both dimensions, and the momentum distribution was recorded periodically to assure that it remained symmetric (and gaussian). The USB and LSB phase and amplitude responses were measured at the $\mathrm{N}=16,17,18,19$ harmonics $(9.6-12.4 \mathrm{MHz})$ and an average $\phi_{U S B}-\phi_{L S B}$ was used in the analysis. The phase differences between adjacent USB's and adjacent LSB's was used to calculate and correct for any additional local electronic phase slope (set $\left.\omega_{c}=\omega_{0}\right)$. A typical measurement of a single sideband is shown in Figure 2.
The phase advance to 48 horizontal pickups and 42 vertical pickups was measured.

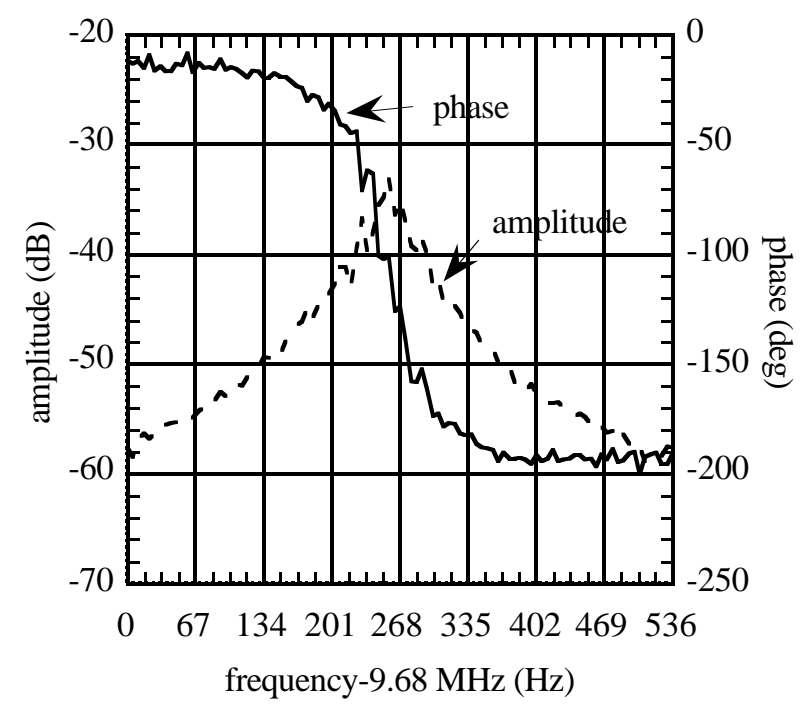

Figure 2: Typical sideband measurement. Network analyzer parameters were: ResBW $=10 \mathrm{~Hz}$, Power $=-26 \mathrm{~dB}$, sweep=20 sec, \#pts=101, \#avg=1.

\section{ANALYSIS}

Figure 3 is a plot of the difference between the measured phase advance from location $\mathrm{A} 1 \mathrm{Q} 1$ to each pickup and the calculated phase advance based on the TevI design. A1Q1 is $7 \mathrm{~m}$ from the center of a zero dispersion straight section which is a point of symmetry in the ring. There are several sources of error in the phase measurements. 1) The error due to the finite signal/noise ratio is approximately 2 degrees, as determined by observation of repeated measurements.

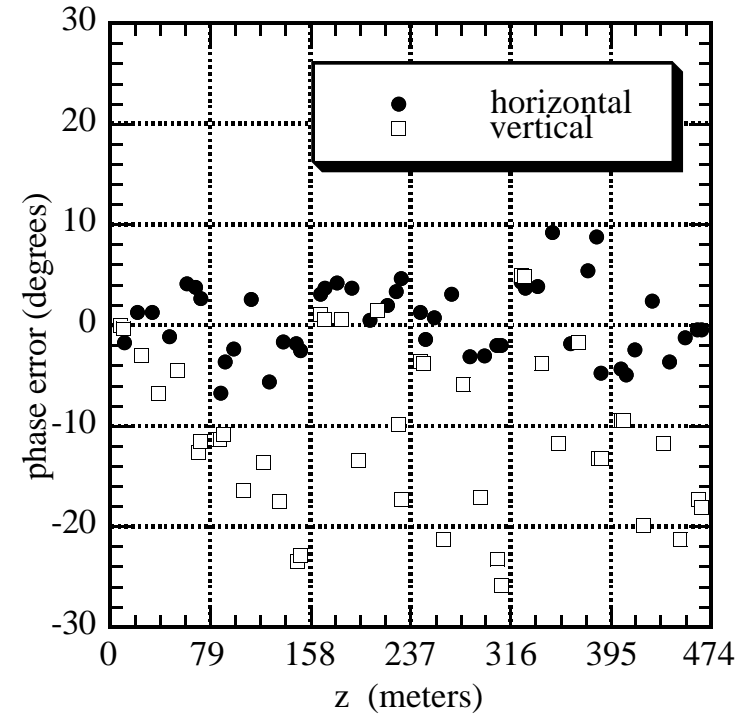

Figure 3: Difference between measured phase advance and phase advance calculated from TevI model. 
2) The error due to the nonlinearity of the electronic phase slope is measured to be $\leq 1$ degree. 3) The error due to the excitation disturbing the beam and distorting the theoretical response was kept to $\leq 2$ degrees by keeping the excitation amplitude as small as possible. The errors due to an asymmetry in the beam distribution and finite chromaticity were calculated from eq (1) and found to be negligible in this case.

Quadrupole, sextupole, and octupole gradients were adjusted in the model in an attempt to bring the model into agreement with the measurements. Three techniques were used: 1) Individual quadrupole strengths were adjusted one-by-one to minimize the sum of the squares of the phase deviations. Seven quadrupoles were adjusted by $.1 \%-.5 \%$, introducing a slight asymmetry into the lattice. 2) The four major quadrupole busses were varied, and the average strength of each quadrupole type was adjusted by $.0 \%-.2 \%$. 3) A global linear least square fit was done for the 12 quadrupole gradients and bus currents to minimize the sum of the squares of the phase deviations. This last method was found to be ineffective. The phase deviations between measurement and model, after these adjustments, is shown in Figure 4. The vertical phase deviations have shifted to be approximately symmetric about 0 degrees, and the maximum swing has been reduced by about $20 \%$. However, there is still a systematic difference between measurement and model for the vertical measurements. Figure 5 shows the vertical beta function in $1 / 3$ of the ring as predicted by the adjusted model.

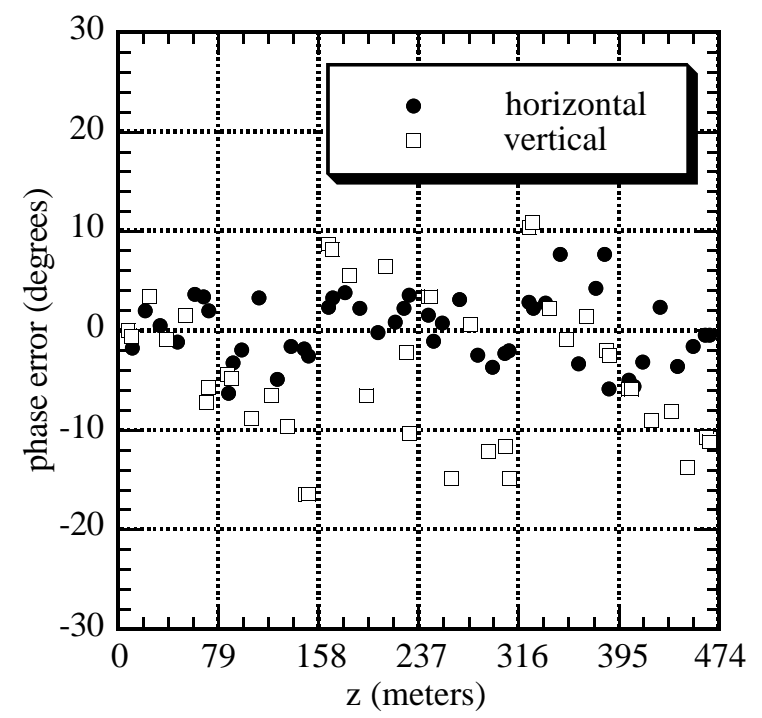

Figure 4: Difference between measured phase advance and phase advance calculated from adjusted model.

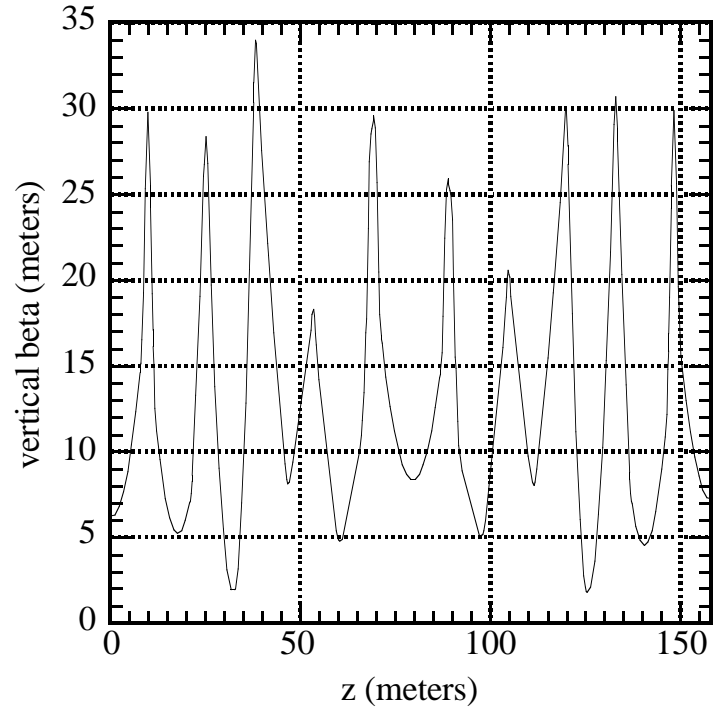

Figure 5: Vertical beta function of adjusted model. Asymmetries and differences from TevI model are approximately $5 \%$.

\section{CONCLUSIONS}

The measurements made here have some agreement with the model, although there is clearly a discrepancy in the vertical case. The source of this discrepancy is unknown. The type of measurement described here requires that cables be run from pickups and kickers back to a single network analyzer, so it is practically limited to storage rings of small to modest size. In addition, an approximate model of the lattice is required at the outset in order to make some sense of the data. This technique offers an alternative to the more usual technique of measuring the phase advance by turn-by-turn oscillations due to a one-pass kick to the beam. The two techniques are in many ways equivalent, although this author has not fully investigated this equivalence.

\section{REFERENCES}

[1] "Methodical Accelerator Design,"Version 8.13/8 CERN/SL/9013(AP) (1990)

[2] Design Report for the Tevatron I Project, Fermilab (1984)

[3] S van der Meer, "A Different Formulation of the Longitudinal and Transverse Beam Response", CERN/PS/AA/80-4, (1980)

[4] J Marriner, D McGinnis, "An Introduction to Stochastic Cooling", in AIP Conference Proceedings \#249, ed. M Month, M Dienes, New York (1992)

[5] S Holmes, et al, "The Tev1 Beam Position Monitor System" IEEE Trans. Nucl. Sci. NS-28 \#3, p2323 (1981)

[6] J Petter, et al, "Transverse Beam Dampers for the FNAL Antiproton Rings", in IEEE 1987 Part. Acc. Conf. V2, p791, Washington DC (1987) 